\title{
The Explanatory Power of Explanatory Variables
}

\begin{abstract}
This paper concerns potential disparities between narratives and statistical evidence in empirical accounting research. We focus on the extent to which a regression model's main variable of interest contributes incrementally to the explanation of the dependent variable. We replicate ten recently published accounting studies, all of which base their conclusions on t-statistics and statistical significance. In eight of the replicated studies, we find that the incremental explanatory power contributed by the main variable of interest is effectively zero. For the remaining two, the incremental contribution is at best marginal. These findings highlight the apparent overreliance on t-statistics as the primary evaluation metric. T-statistics tend to reject the null hypothesis primarily due to a large numbers of observations $(\mathrm{N})$, a point we examine in detail. As a potential remedy, we evaluate the use of Standardized Regressions (SR). The magnitudes of estimated SR coefficients indicate variables' relevance directly. Empirical analyses establish a strong correlation between a variable's estimated SR coefficient magnitude and its incremental explanatory power, without reference to $\mathrm{N}$ or t-statistics.
\end{abstract}

Keywords: explanatory power, classical statistics, large N, standardized regressions. 


\section{Introduction}

Contemporary empirical accounting research often relies on regression analyses with three classes of independent variables: fixed effects (years, firms and/or industries), regular control variables (typically motivated by prior literature), and, most important, a main variable of interest (sometimes complemented by interactive terms). With few exceptions, findings related to the estimated regressions report that (i) the main variable of interest has the correct sign, and (ii) the t-statistic rejects the null at conventional levels. Researchers generally view such evidence as adequate to propose that the hypothesized story reflects the real world. To be sure, studies typically include a battery of supplementary tests, such as the evaluation of multiple specifications with varying constructs and measurements, robustness tests, and expanded analyses addressing endogeneity issues. Researchers rarely, if ever, suggest that these tests overturn the overall conclusions: most papers tend to view a "correct sign" and a large enough t-statistic as what primarily needs to be achieved. This modus operandi has gained considerable status as a major, if not the major, empirical accounting research paradigm. ${ }^{1}$

Most researchers are aware that a significant t-statistic can mislead if the OLS regression violates standard assumptions (e.g. homoscedasticity and uncorrelated error terms), and, as a matter of routine, adjust t-statistics for clustering. That said, a much more fundamental issue is rarely addressed: even when measured accurately, a t-statistic by itself does not connect directly with the extent to which a variable contributes to the explanation of the dependent variable. The absent connection becomes most obvious, and inescapable, when the number of observations, $\mathrm{N}$, is large.

\footnotetext{
${ }^{1}$ The current research paradigm is often attributed to Petersen (2009), who argues that it is powerful and preferable to traditional Fama-MacBeth regressions which eschew year fixed effects. Regardless of implementation details, it should be stressed that the paradigm centers on t-statistics. Further, research applying logit/probit rather than OLS regressions effectively work much the same.
} 
A t-statistic of 2 will not impress unless $\mathrm{N}$ is sufficiently small; conversely, experienced researchers may subjectively assess that 2 is not sufficiently large if $\mathrm{N}$ equals 50,000 , for example.

The statistics literature makes it clear that the rejection of a null hypothesis by itself "is not informative in large samples" (see Abadie (2020), p. 198 for an extensive analysis. See also, McShane et al. (2019) and Gannon et al. (2019) who argue for N-dependent significance tests). The notion of "large samples" is open ended and subjective, so the extent of an independent variable's t-statistic informativeness becomes an empirical question. An improved understanding must, therefore, consider alternative metrics of a variable's incremental explanatory power. This simple observation underpins the focus and contribution of this paper.

As a strictly practical matter, the issue of incremental explanatory power can be easily addressed by calculating the decrease in explanatory power (commonly measured by $\mathrm{R}^{2}$ ) after having deleted the relevant right-hand-side variable. No matter the t-statistic, the change may be minuscule (e.g. equivalence in the first three digits). One can argue that in such cases, it is misleading to claim that a significant t-statistic implies that the variable of interest explains (or connects with, relates to, associates with) the dependent variable. Under normal circumstances, a significant t-statistic is a necessary but insufficient condition for a variable to contribute to the explanation of the dependent variable. $^{2}$

To elaborate on the last paragraph, note that a comparative analysis of t-statistics across independent variables in a specific regression can potentially indicate the relative incremental explanatory power of these variables. This is a separate issue, though: $t$-statistics are of no direct interest in assessing magnitudes of incremental explanatory power attributable to each independent

\footnotetext{
2 To be sure, "full" p-values offer no improvement over t-statistics in this regard. P-values accompanied by inequality signs are actually worse than t-statistics, since information needed by the reader to assess materiality is lost.
} 
variable. The ranking of t-statistics extracted from a specific regression is at best only ordinal (and hence of no apparent relevance across different regression specifications).

This paper examines 10 accounting research papers to determine, for each study, the extent to which the independent main variable of interest ("MVI") contributes to explaining the dependent variable. The studies have been sampled pseudo-randomly from top accounting journals. Our motivation for this stems from the idea that the issue of overreliance on t-statistics and disparities between narratives and statistical evidence may be more difficult to dismiss if it can be shown to exist in published work. Replicating published work also provides an interesting angle insofar that authors are motivated to claim that the MVI is relevant- that is, the desired outcome is null hypothesis rejection. All replicated papers apply the research paradigm discussed above and, crucially, identify one key MVI. Our empirical analysis centers on what we view as each study's main regression. We aim at understanding what the 10 identified main regressions have in common, and how they vary. To be sure, we in no way assess the replicated studies' degree of overall validity. To avoid detracting from the issues focused on in this paper, all studies have been anonymized to prevent the underlying papers from being identified.

Our empirical analysis comprises four parts. The first part is central: we calculate the incremental explanatory power attributable to the MVI. The results are then juxtaposed to various alternative correlation statistics. The second part of the empirical analysis addresses whether the 10 replicated regressions only require a small number of independent variables to effectively explain the dependent variable, and, further, whether the MVI belongs to this set of substantive variables. The third part concerns the informativeness of t-statistics in light of their dependence on $\mathrm{N}$. The fourth and final part of our empirical analysis pertains to the potential efficacy of Standardized Regressions (SR). Standardized Regressions shift the analysis away from t-statistics and toward 
the magnitude of the estimated coefficients, and as such, they can potentially improve on research practice. We analyze whether relatively low (high) SR coefficients are likely to yield low (high) variable relevance, as measured by incremental explanatory power.

We summarize our findings as follows:

1. The incremental explanatory power of the MVI is, overall, at best marginal. In five out of ten replicated studies, the inclusion of the MVI in the regression increases the explanatory power (measured as the Pearson correlation between the dependent variable and the modelprojected dependent variable, the square root of $R^{2}$ ) by less than 0.0005 . For all ten studies, the maximum increase is 0.004 . These results suggest a disparity between the narrative of the papers we have examined and the empirical evidence presented in the key regression: none of the papers examined can refer to the main regression as providing substantive support for their conclusions.

2. In all studies, no more than five variables effectively explain the dependent variable. These variables have relatively large t-statistics, and they always exceed the ones related to the MVIs. This finding aligns with the first point: adding the MVI to the regressions' righthand side does not meaningfully improve the models' explanatory power. The fact that the MVIs' t-statistics all show statistical significance does not contradict this conclusion.

3. The significant $t$-statistics of the MVIs can generally be attributed to the large sample sizes. Analyses show that to achieve a t-statistic of 2 , the minimum required sample size exceeds 10,000 observations in four cases; the remaining six require approximately 2,000 to 10,000 observations. As a crude diagnostic, about half the studies will no longer achieve statistical significance if the sample size had been less than half of the actual sample size. 
4. Results related to Standardized Regressions show that the magnitude of estimated coefficients correlates strongly with variables' incremental explanatory power, with a rank correlation of 0.9 . We propose the following rule of thumb: if the estimated SR coefficient is 0.05 or less, then the incremental explanatory power is no more than 0.001 . Of the ten MVIs, all but two fall below this threshold.

It can reasonably be argued that these findings do not surprise. The current research paradigm has existed for many years, and hundreds of published papers are rooted in it. Accordingly, the paradigm comes with a collective "researchers' experience per folklore". As far as we can tell, some of this folklore does suggest that MVIs all too often can be viewed as irrelevant even though their t-values are large enough to reject the null hypothesis. This folklore goes beyond mere suspicion: in the past, many studies did report changes in $\mathrm{R}^{2}$ attributable to the MVI. These findings rarely received comments, as if such outcomes were irrelevant. We surmise that less-thansupportive outcomes caused this reporting practice to subsequently vanish. A contribution of this paper is that our evidence suggests there are good reasons why this old-fashioned reporting practice should be restored, as part of a full disclosure convention.

There is also no doubt that the research community is aware that traditional significance levels ought to be adjusted to raise the bar to reject the null hypothesis as $\mathrm{N}$ becomes very large. But no objective, agreed-upon procedures have ever been suggested, let alone materialized. In the absence of an objective adjustment procedure, subjectivity in how to interpret statistical evidence by both authors and readers of research becomes important and should be recognized—and not be comingled with objectivity or sufficiency. This point highlights why a singular focus on t-statistics falls short. 


\section{Context and Background}

In broad terms, the empirical part of this paper addresses whether data analyses found in the literature reject null hypotheses even though it could be argued that the statistical evidence is neither compelling nor material. ${ }^{3}$ In a traditional regression context, the issue pertains to whether MVIs actually help to explain the dependent variables. Until a few decades ago, researchers often dealt with this issue by relying on the Fama-MacBeth method (Fama and MacBeth (1973)). This method typically estimates annual regressions and subsequently calculates the relative frequency of "correct signs" associated with variable coefficients across years (a refined and more formal version tests whether t-statistics associated with the average coefficient across years differs from zero). Anecdotally, the Fama-MacBeth method earned a reputation of excessively failing to reject the null hypothesis. At least to some extent, this can be attributed to a lack of statistical power; the number of years in a study typically determines the number of observations, and this number tends to be small (rarely exceeding 50). As a consequence, the Fama-MacBeth method may lead to false — and arguably too frequent—-failures to reject the null hypothesis (i.e. Type II errors).

Contemporary empirical accounting research differs in its modus operandi. In addition to an MVI and regular controlling variables (commonly motivated by prior literature), regressions commonly include fixed effects (for years, firms and/or industries): these justify a pooling of data across strata. The resulting regressions can hence make use of very large numbers of observations. Given t-statistics' reliance on $\mathrm{N}$, these regressions therefore produce an abundance of significant variables, even after adjusting for clustering. To the critical reader, this abundance of significant

\footnotetext{
${ }^{3}$ A prominent example of how the finance literature deals with this issue is Harvey's presidential address (2017). See also Harvey, Liu, and Zhu (2016); Chordia, Goyal, and Saretto (2017). Some papers in economics and finance also suggest that research becomes all too often biased due to publication incentives and that there are traces in the published tables. See for example Brodeur, Sangnier, and Zylberberg (2016); Moosa (2017); Dyckman and Zeff (2015).
} 
variables is neither unsurprising nor particularly informative. As noted earlier, significant tstatistics alone are far from sufficient to judge whether a variable makes a substantive difference in a regression specification. The change from the Fama-MacBeth method to the contemporary fixed-effects-based method hence implies that false null rejections have replaced false failures to reject the null as a major concern.

The potential excess of false null rejections in contemporary empirical accounting research suggests that it may be worthwhile to evaluate the possibility that proposed MVIs all too often are, in fact, not as relevant as the related narrative may suggest. Sharply put, if an MVI is deleted from a regression's right-hand side, the reconstituted right-hand side may not result in a materially different level of explanatory power. In such cases, can one really claim that the MVI is relevant in any practical sense? This is a subjective issue, but in our view, such subjectivity cannot—should not-be avoided.

There is a vast literature on the topic of variables' degree of explanatory power (or degree of materiality) in statistics and economics (c.f. Ioannidis 2005; Ioannidis, Stanley, and Doucouliagos. 2017; Andrews, Isaiah, and Kasy 2019; Gelman, Andrew, and Stern 2006). The accounting literature includes Cready et al. (2020), Dyckman and Zeff (2014, 2015); Dyckman (2016); Leone, Minutti-Meza, and Wasley (2019); Kim, Ji, and Ahmed (2018); Hail, Lang, and Leuz (2020); Black et al (2019); Ohlson $(2015,2020)$ and Biddle, Seow and Siegel (1995). Although these papers tackle the same broad issue as we do, it should be noted that they also focus on numerous sub-topics that will not be raised in this paper (e.g. "screen picking", the role of specification, winsorization and trimming, procedures to avoid sample biases, how to deal with null hypotheses as opposed to alternative hypotheses, the so-called replicability issue, the mathematics of tstatistics and potential comparability issues, classical vs. Bayesian statistics, etc.). Instead, 
referring only to easily understood first principles of data analysis, this paper addresses issues relevant to researchers and readers of research for assessing the materiality of statistical evidence and the extent to which a proposed narrative is supported by the data.

One such first principle relates to the number of variables required to effectively explain the dependent variable. This issue involves both experience and judgement. When computers first became part of the researcher's toolset, the collective experience suggested that a few carefully selected variables should suffice. If the claim has merit, researchers should be aware that introducing another variable beyond the traditional, well-established controlling variables is unlikely to make a material difference, in terms of explanatory power. The common practice of employing as many as 15 or more variables on the regression's right-hand side (not counting fixed effects) may look more like data-dredging than thoughtful diligence.

We also make a few additional comments here concerning t-statistics and large sample sizes. A subtle and seldom recognized issue is that unless the true value of a coefficient is exactly zero, a significant t-statistic lacks information content when the number of observations is very large (Abadie (2020)). ${ }^{4,5}$ A famous manifestation of this is the Jeffreys-Lindley paradox (roughly that false null hypothesis rejection is guaranteed when $\mathrm{N}$ goes to infinity; see Lindley (2014) and Spanos (2003) for an extensive discussion). Consistent with intuition, Leamer (1978) shows that standard rationality assumptions imply that the use of t-values must depend on N: "the significance level should consequently be a decreasing level of the sample size" (Leamer (1978), italics per author). T-statistics adjusted for $\mathrm{N}$ have never come into practice, seemingly because it would be difficult to establish conventions as to the appropriate cut-off levels for making claims about the

\footnotetext{
${ }^{4}$ Pearson himself suggested more than a hundred years ago that significance cut-offs ought to be adjusted as the sample size increases.

${ }^{5}$ Abadie (2020) argues that the cases of non-significance are actually more informative.
} 
degree of significance. Nonetheless, as noted above, many researchers, if not the overwhelming majority, intuitively know that a t-statistic of 2 with $\mathrm{N}>50,000$ de facto implies a less-thanimpressive significance level.

Finally, we make a few comments on Standardized Regressions (SR). As the name suggests, such regressions standardize dependent and independent variables to ensure that these satisfy unit standard deviation. ${ }^{6}$ The advantage with SR pertains to the comparability of estimated coefficients. Because of the standardization, coefficients act as normalized elasticities; across the independent variables, the greater the coefficient, the more it influences the dependent variable (c.f. Affifi and Clarke 1990; Darlington 1990; Walsh 1990). Importantly, SR coefficients are completely independent of $\mathrm{N}$ (and t-statistics).

In the absence of multicollinearity, an estimated SR coefficient equals the simple correlation between the independent variable and the dependent variable (Bring (1994)). It is hence easily interpretable (e.g. since most people would agree that a correlation of 0.1 is immaterial, the same could be said of an SR coefficient of the same magnitude). ${ }^{7}$ In the presence of multicollinearity, the one-to-one mapping of SR coefficient size to incremental explanatory power is no longer present (Bring (1994)). The informativeness of SR coefficients must then be evaluated empirically. We do this using data and variables from the ten studies whose main regression we replicate. Since the interpretation of SR coefficients is not regression specific, we can then proceed to evaluate whether a global cut-off point can act as a rule of thumb for materiality. It is this broad cross-

\footnotetext{
${ }^{6}$ Examples of accounting studies that use standardized regressions including Hirst 1983; Ferreira and Matos 2008; Shan, Taylor, and Walter 2014.

${ }^{7}$ A bivariate correlation of 0.1 is visually indiscernible to the eye in a scatterplot.
} 
sectional perspective on Standardized Regressions that we view as particularly worthwhile to investigate. From what we can tell, this has never been considered in the accounting literature.

\section{Selection of Studies and Evaluation of Replicability}

\subsection{Studies Replicated: Selection}

To make our research as relevant and interesting as possible, we replicated regressions from recent studies. We pseudo-randomly chose papers published in four out of the five highest-ranking accounting journals between 2014 and $2017 .{ }^{8}$ We initially screened the total pool of potential candidate papers via their respective abstracts to filter out papers that do not rely on empirical analyses. For the remaining papers, we carefully read the empirical analysis sections to ensure that all data on which the main tables are based are available in commonly used databases, such as Compustat, I/B/E/S, and CRSP. In addition, each study must (i) identify one MVI on which the narrative centers, (ii) identify a table of apparent main interest, (iii) provide a clear description of the regression variables' construction, and (iv) provide distributions and correlations of the variables used. Finally, we also required that the studies rely on at least 10,000 observations and one set of fixed effects. We do not impose any selection criteria regarding research questions or topics. This process leads to eleven papers subject to replication attempts.

For each paper, our procedures strictly followed the recipe for variable creation. To assess the degree of success, we compared descriptive statistics for our variables to those of the original studies. We required the signs and the first non-zero digit of each variable's median, mean, and

\footnotetext{
${ }^{8}$ Note that we at no point assess or discuss the frequency with which the issues this paper concerns arise in the accounting literature. The papers selected are not claimed to be representative for all empirical accounting research. Having that said, the fact that we could easily obtain ten recent papers from top journals at least suggests that the points we raise merit discussion.
} 
standard deviation to closely parallel those in the original study. If this criterion fell short, we relied on correlation matrices to evaluate alternative interpretations of how variables were specified in the original studies and also approached the authors for guidance to eliminate differences to the fullest extent possible. A final diagnostic pertains to the regression results in what we judged to be each paper's main table. Our regression results were compared to those in the original papers. If significant variables in the original studies were not significant in our replications, or had incorrect signs, we revisited the variable creation process and explored alternative interpretations of the description for the problematic variable in question. After these procedures, 241 of 245 significant variables across all studies had the same sign as in the original studies. Only one paper was dropped; we failed in this case to obtain variable distributions that approximated those of the original paper. Our final sample therefore comprises main regressions from ten studies, all of which for all practical purposes can be viewed as replications.

\section{Table 1. Characterization of the ten original studies}

Descriptive statistics ( $\mathrm{N}$, adjusted $\mathrm{R}^{2}$ and $\mathrm{t}$-statistics) extracted from the original studies are tabulated in Table 1. All variables are anonymized and denoted Varl - Var31 according to the descending order of the absolute value of their respective t-statistics. The main variables of interest are indicated in bold-face. The number of independent variables across studies vary from 11 to 31 and the adjusted $\mathrm{R}^{2}$ varies from 0.035 to 0.867 . The sample sizes range from 14,920 to $2,471,917$ observations.

\subsection{Success of replication}

As indicated above, to evaluate the extent of replication success, we focus on $\mathrm{N}$, adjusted $\mathrm{R}^{2}$ and t-statistics. Results are shown in Table 2. 


\section{Table 2. Success of the ten replications}

With respect to sample size, the percentage difference between our replication samples and the original papers' samples $\left(N\right.$ Diff) has a very narrow range, $-5.4 \%$ to $4.6 \%$. ${ }^{9}$ The difference in adjusted $\mathrm{R}^{2}$ between our replications and the original regressions (Adj. $R^{2}$ Diff) is also narrow, ranging from -0.062 to 0.027 , with an average of -0.012 . Turning next to t-statistics, Table 2 juxtaposed to Table 1 shows that, overall, the t-statistics for our replicated studies are very similar to those in the original studies. As noted above, the vast majority of significant variables have coefficients with the same sign as in the original study (241 of 245). In seven replications, sign equivalence is true for every significant variable. In two replications, one significant variable shows the opposite sign and in one replication there are two opposite signs. With respect to the MVIs, our replications consistently yield significance levels that approximate those in the original studies, with an average difference of $-0.4 \%$.

\section{Empirical Analyses}

\section{1 - Overview}

The primary purpose of this empirical section is to assess variable materiality. This inevitably involves our using subjective language, and we urge readers to scrutinize tables and results to assess whether our judgements are reasonable.

Before reporting on findings, we outline again the four main parts of issues addressed, along with notable subparts. First, as a precursor, we calculate the correlation between the MVIs and the dependent variables. We then proceed to focus on the extent to which the main variable of interest

\footnotetext{
${ }^{9}$ Databases regularly correct and update historical data, which may be a potential reason for the difference in sample sizes.
} 
actually contributes to explaining the dependent variable in the full regression; this includes all the controlling variables. The methodology is straightforward: we compare the explanatory power of two regression specifications, with and without the MVI to determine the MVIs' incremental explanatory power. Second, we evaluate the number of variables needed to effectively explain the dependent variables. We then investigate whether the MVIs are part of this set of variables. Third, we assess the role of t-statistics and the extent to which these can serve as indicators of the independent variables' explanatory power. In this context, we consider the empirical implications of large N. Finally, we evaluate Standardized Regression coefficient estimates and whether these re-scaled coefficients inform on the independent variables' incremental explanatory power.

\section{2 - Preliminary Correlations: Main Variable of Interest (MVI) and Dependent Variable.}

The following question provides a natural starting point for the empirical analysis: to what extent does the MVI correlate with the dependent variable absent intricacies related to multicollinearity? If an MVI does not correlate (or has a low correlation) with the dependent variable, one would be obliged to acknowledge that including additional variables in the regression specification could tilt either for or against null hypothesis rejection. By contrast, a non-trivial correlation between the MVI and the dependent variable would provide prima facie evidence that one could proceed to more elaborate analysis, inclusive of controlling variables (which, of course, can render the MVI insignificant). This kind of simplistic and precursory analysis yields a better understanding of how to explain the dependent variable and shows if the final regression results are, at least in part, a consequence of multicollinearity.

Table 3. Correlations: main variables of interest and dependent variables 
For each of our replicated studies, Table 3 shows Pearson and Spearman correlations between the MVI and the dependent variable ( $P$. Corr. and $S$. Corr, respectively). Rather than just restating the numbers in the table, we here summarize and interpret our findings. When the absolute value of a correlation is in the range of 0.0 to 0.1 , we consider the correlation to be negligible (informally, plotting such data would not show any correlation discernable to the eye). The absolute value of correlations in the $0.1-0.2$ range we view as being on the weak side (a scatterplot would provide unpersuasive evidence), correlations with absolute values between 0.2 and 0.3 imply a moderate correlation (a scatterplot would show a discernable correlation), and, finally, if the absolute value of a correlation is above 0.3 , we deem the correlation strong (a scatterplot would show a distinct correlation). Given this nomenclature, six of our ten sample studies show negligible correlations between the MVI and the dependent variable, two show weak correlations, and, for the two remaining, one is moderate and one is strong. In our view, the overall evidence suggests that the replicated studies typically have a weak foundation on which to build a case that the MVI in some way connects with the dependent variable. This view involves unescapable subjectivity, and reasonable people can disagree about materiality.

All of the replicated regressions include fixed effects and one can hence argue that the above correlation analysis is not fair unless one first adjusts for these. To address this matter, we consider two regression specifications inclusive of fixed effects (as per each respective original study): one with and one without the MVI as the only other independent variable.

\section{Table 4. Correlations: main variables of interest, fixed effects and dependent variables}

Pearson correlations between the dependent variable and fitted values based solely on fixed effects are denoted FE bivariate. Similarly, correlations between the dependent variable and fitted values 
based on fixed effects plus the MVI are denoted FE+MVI bivariate. Chg. in Corr denotes the difference in Pearson correlation between these two specifications and hence the incremental effect due to the inclusion of the MVI.

Table 4 shows that the FE bivariate correlations range from 0.087 to 0.551 and hence that fixed effects do make a difference as an explanatory factor: they obviously contribute more explanatory power than the MVI. FE+MVI bivariate correlations range from 0.131 to 0.624 , which of course exceeds the FE bivariate correlations. In terms of improvements across studies, Chg. in Corr shows a minimum of 0.000 and a maximum 0.118. Specifically, for three studies, Chg. in Corr is 0.000; for five studies, Chg. in Corr ranges from 0.001 to 0.1; and for two studies, Chg. in Corr is between 0.1 and 0.118. Compared to the Pearson correlations in Table 3, Chg. in Corr are always smaller in absolute value terms, leading us to the following conclusion: overall, inclusion of fixed effects reduces the MVIs' incremental explanatory power. This finding is by no means foregone, and it is a less than encouraging sign of what to expect from a regression specification that also includes controlling variables.

We next approach the issue of incremental explanatory power of the MVI from a slightly different angle: we control for the non-MVI independent variables but leave out effects due to multicollinearity between the MVI and these variables. Concretely, we correlate the MVI with residuals extracted from the baseline regression — the regression which includes fixed effect and controlling variables, but excludes the MVI. These correlations indicate the extent to which variation not captured by the baseline model is captured by the MVI, without exploiting potential advantages associated with multicollinearity. Low correlations would indicate that the MVI can only contribute incrementally as an explanatory variable if it correlates with one or more of the controlling variables. In other words, multicollinearity would in such cases be a necessary but 
insufficient condition for statistical significance. To elaborate, if the correlations in this test are low, one would have to invoke multicollinearity arguments and/or explicit or implicit claims regarding inadequate regression specifications in the prior literature.

\section{Table 5. Correlations: Main variables of interest and residuals from the baseline models}

The results from this test, shown in Table 5, provide a blunt message: none of the ten studies show (absolute value) correlations larger than 0.1. In fact, correlations are lower than 0.05 for eight studies. A scatterplot with variation left unexplained by the baseline model (the residuals) on one axis and the MVI on the other would hence not show any correlation discernable to the eye.

\section{3 - Incremental Explanatory Power of MVIS}

We next conduct the most poignant test so far. It aims to answer the central question: if one deletes the MVI, to what extent is the regression model's explanatory power affected? On a basic level, the issue pertains to a change in goodness-of-fit and, if OLS is applied, alternatives to Pearson correlations ought to be considered. Pearson correlations (and $\mathrm{R}^{2}$ ) are sensitive to overfitting since the OLS estimation method is directly tied to the evaluation metric: adding an independent variable always increases such correlations (and $\mathrm{R}^{2}$ ). We therefore also consider Spearman correlations as an independent evaluation metric. To appreciate the role of this metric, note that it allows for a decrease in explanatory power after an addition of the MVI, unlike Pearson correlations (and $\mathrm{R}^{2}$ ). ${ }^{10}$

\section{Table 6. Incremental explanatory power of the main variables of interest}

Table 6 reports the goodness-of-fit metrics (Pearson and Spearman correlations) for the two sets of regressions we compare: first, the correlation between the dependent variable and fitted values

\footnotetext{
${ }^{10}$ Using adjusted $\mathrm{R}^{2}$ does not invalidate this point. Adjusted $\mathrm{R}^{2}$ and unadjusted $\mathrm{R}^{2}$ are highly unlikely to differ on the first four digits when $\mathrm{N}>10,000$.
} 
of the baseline regression specification (which excludes the MVI), and, second, the correlation between the dependent variable and fitted values of the full model (which includes the MVI). The maximum Chg. in P. Corr. is 0.004 , which, in our judgement, is markedly small. For five studies, Chg. in P. Corr. is 0.000. This finding makes it difficult, if not impossible, to claim that the MVIs incrementally explain the dependent variable in these studies. Turning to the Spearman correlations, one study actually exhibits a decrease in explanatory power when including the MVI in the regression specification. Having that said, the case is somewhat idiosyncratic; Table 6 shows that, for all practical purposes, the differences between Spearman and Pearson correlations are trite.

It is worth repeating that although Table 6 shows that changes in goodness-of-fit due to the MVIs are very small, all MVIs have t-statistics larger than 2 (as was shown in Table 2 and in the original studies). This motivates an evaluation of the extent to which t-statistics bear on a variable's incremental explanatory power.

\section{4 - Incremental Explanatory Power and T-statistics}

We begin this subsection by hypothesizing that t-statistics' intra-study relative magnitudes bear on variables' relative incremental explanatory power. Specifically, for each study, separately, we rank all variables in terms of absolute t-statistics, from highest to lowest (including insignificant tstatics), and then evaluate whether overall explanatory power increases - but at a decreasing rateas variables are sequentially added to the regression specification. We also evaluate the number of independent variables required to reach an effective maximum explanatory power, such that additional independent variables only provide trivial incremental increases. Broadly speaking, an interesting finding would be if the great majority of the studies whose main regression we replicate only require a small number of variables to effectively explain the dependent variable. 
We evaluate whether the adding of the MVI occurs before or after the effective maximum explanatory power is reached. Given the empirical results so far, we hypothesize that the MVIs do not fall into the set of variables that make a substantive difference.

\section{Figure 1. Incremental explanatory power by t-statistic (Pearson)}

Figure 1 plots Pearson correlations between dependent variables and fitted values per estimated models against the number of independent variables in the regression specification. For each study, the regression specification behind the left-most datapoint in Figure 1 only includes fixed effects and the independent variable with the largest t-statistic in the full regression. Independent variables are sequentially added to the regression specification, based on their respective absolute t-statistics. This sequential treatment of variables does not distinguish between MVIs and regular controlling variables.

As Figure 1 shows, while the Pearson correlations increase with the adding of independent variables, the marginal increase quickly becomes small: in half of the cases, there is no visually discernable increase in explanatory power after five independent variables have been added to the regression specification. For the other half, the same phenomenon occurs after only three independent variables have been included. With respect to the MVIs, their rankings are higher than 5 for all studies and the average ranking is 11.2. These results suggest that the MVIs cannot in any reasonable sense be expected to meaningfully contribute to the explanation of the dependent variable.

\section{Figure 2. Incremental explanatory power by t-statistic (Spearman)}

Results based on Spearman correlations, shown in Figure 2, closely resemble those in Figure 1. The main difference is that variables with low t-statistics can now visibly reduce a model's 
explanatory power, a phenomenon that occurs for three of the studies. It serves as a reminder that in regression modelling, the adding of independent variables is not cost-free. Any variable contributes both noise and information content, and at some point, the noise becomes dominating, resulting in negative incremental explanatory power.

To summarize, Figures 1 and 2 make it clear that anchoring regression specifications on a small set of well-stablished independent variables leads to a model that cannot easily be improved on, at least not in the ten cases we have investigated. The absence of MVIs in the set of relevant variables reinforces our previous findings: the ten MVIs do not contribute meaningfully to the explanation of the ten dependent variables.

\section{5 -Large N}

This subsection concerns a question which so far has been making its presence felt only indirectly: what are the implications of a large N? Empirical accounting research typically does not discuss N's magnitude and how N may influence interpretations of findings and associated narratives; instead, the literature tends to solely focus on t-statistic or p-values. We argue that the issue of large $\mathrm{N}$ must be dealt with.

As noted earlier, statistical significance is entirely consistent with variables which effectively contributes nothing to the explanation of the dependent variable if $\mathrm{N}$ is sufficiently large. ${ }^{11}$ This is clear from the approximation formula $t=\operatorname{sqrt}(N) \times K(N)$, where standard theory (c.f. Greene 2003) shows that $\mathrm{K}(\mathrm{N})$ does not depend on $\mathrm{N}$ as $\mathrm{N}$ becomes large. An instructive special case shows that

\footnotetext{
11 Appendix 1 illustrates this point. For all replicated studies, we augment the full regression specification with variables that capture the maximum temperature, minimum temperature, total precipitation and total snowfall in Denver at the day of the release of the annual report. In four out of ten regressions, at least one of these variables has statistical significance at the $10 \%$-level or better. One can argue that most people would not view these variables as relevant for the explanation of accounting constructs. The absence of a compelling narrative makes the statistical evidence easily dismissible. One can further argue that if a compelling narrative does exist, this should not influence researchers' evaluation of the merits of the actual statistical evidence.
} 
if the simple correlation between the dependent and independent variables is relatively small, $\mathrm{K}(\mathrm{N})$ approximates the asymptotic correlation. Due to the $\operatorname{sqrt}(\mathrm{N})$ term, it follows that modest but significant $\mathrm{t}$-statistics do not impress when $\mathrm{N}$ is large and at most provide incomplete evidence regarding a variable's relevance for explaining the dependent variable. ${ }^{12}$ It is well to note that real-world phenomena do not depend on $\mathrm{N}$, and it therefore helps if researchers provide insights that do not depend directly on $\mathrm{N}$ either. In this regard, the formula $t=\operatorname{sqrt}(\mathrm{N}) \times \mathrm{K}$ imposes mental discipline.

We evaluate the importance of $\mathrm{N}$ using a simplistic and intuitive diagnostic, which can be applied in all empirical research. This diagnostic estimates the number of observations required to obtain a t-statistic that approximates 2 . If a very large number of observations is needed to get a modest t-statistic of 2, the variable should perhaps be dismissed as irrelevant. The term "very large" is of course subjective, which is unavoidable. In our view, the underlying idea that a seemingly unreasonably large $\mathrm{N}$ may be required for statistical significance is not easily dismissed.

To measure the minimum required $\mathrm{N}$, we use two approximation methods: one based on back-ofthe-napkin calculations, and one based on simulations. The critical number of observations needed

\footnotetext{
${ }^{12}$ The issue of large $\mathrm{N}$ renders the correction for clustering largely a moot point. Assuming that the OLS estimation is asymptotically consistent, the claim is apparent since large $\mathrm{N}$ simply implies that one wants statistical significance with some margin to reject the null. The margin required is a subjective matter and in the absence of a correction for clustering one may require a t-statistic of, say, 4 rather than 3 . The enhanced "accuracy" of t-statics that is obtained by correcting for clustering would thus seem to be the least of worries if the issue concerns assertions worthy to be put in an abstract.
} 
to show a t-statistic of approximately 2 obtained using these methods is denoted $\mathrm{N}^{*}$ and $\mathrm{N}^{* *}$, respectively. ${ }^{13}$

The first approximation, $\mathrm{N}^{*}$, derives from the definition of a t-statistic according to classical asymptotic theory:

$$
N^{*}=(2 / t)^{2} \times N
$$

(Equation 1)

$\mathrm{N}$ and $\mathrm{t}$ are obtained from the original studies. $\mathrm{N}^{*}$ can hence always be calculated by readers of research, so long as $\mathrm{N}$ and $\mathrm{t}$ are reported.

Next, consider the approximation $\mathrm{N}^{* *}$. It is based on numerical methods combined with bootstrapping. For each study, we randomly select $n$ observations (without replacement) and run the original regression. The initial $n$ is simply a best guess of $\mathrm{N}^{* *}$, the end result. Using this $n$, the procedure is repeated 100 times, after which the median t-statistic for the MVI is obtained. If the median t-statistic is higher (lower) than 2, we reduce (increase) the subsample size $n$ for another iteration. This procedure is repeated until the median t-statistic for the MVI approximates 2, which yields $\mathrm{N}^{* *}$.

\section{Table 7. $\mathrm{N}^{*}$ and $\mathrm{N}^{* *}$}

\footnotetext{
${ }^{13}$ As an alternative to assessing the effect of $\mathrm{N}$ on the materiality of the variable subject to scrutiny, consider defining $\mathrm{K}=\mathrm{t} / \mathrm{sqrt}(\mathrm{N})$, that is, $\mathrm{K}$ can be interpreted as a " $\mathrm{N}$-normalized $\mathrm{t}$-statistic". It follows immediately that $\mathrm{K}=2 / \mathrm{sqrt}(\mathrm{N} *)$., that is $\mathrm{K}$ and $\operatorname{sqrt}\left(\mathrm{N}^{*}\right)$ is a one-to-one transformation. Thus, if one judges that $\mathrm{N}^{*}=10,000$ makes the variable "immaterial", then the same must be true if $\mathrm{K}=0.02$ (or less). In other words, a K-calculation can be used as a (subjective) diagnostic whether or not an MVI contributes "materially" to the explanation of the dependent variable. (As a comparison, if a researcher views (say) $\mathrm{N}^{*}=5,000$ as a "materiality" cut-off quantity, then the K cut-off comes close to 0.03.) It is noteworthy that the above is simply a matter of common sense. To appreciate what is involved, consider the following. Suppose, in a simple univariate setting, the (sample) mean divided by the standard-deviation equals 0.02 . If someone is asked "is the estimated mean materially different from zero?", many would be inclined to say "no" because, regardless of N, a plot would show a complete scatter (which would not change if the number of observations is doubled). Given a symmetric distribution one can expect about $51 \%$ of the observations to be positive, which at face value suggests a lack of materiality. Of course, the statistical significance depends solely on $\mathrm{N}$; the tstatistic will be 2 or better whenever $\mathrm{N}$ exceeds 10,000 .
} 
Table 7 shows results for the critical number of observations needed. As expected, both $\mathrm{N}^{*}$ and $\mathrm{N}^{* *}$ are smaller than $\mathrm{N}$ for each study (it follows since the reported $\mathrm{t}$-statistics all exceed 2 in the original studies). For a majority of studies, $\mathrm{N}^{*}$ approximates $\mathrm{N}^{* *}$. When there is divergence, the number derived from the back-of-the-napkin method, $\mathrm{N}^{*}$, shows that more observations are required than the simulations suggest.

In four studies, the number of observations required according to both $\mathrm{N}^{*}$ and $\mathrm{N}^{* *}$ is in excess of 10,000. This implies that in a hypothetical world in which the only relevance criterion was a tstatistic above 2 for the MVI, these studies would fall short if the sample instead comprised 10,000 observations or fewer. Out of the remaining studies, five require between 3,000 and 10,000 observations and one study requires approximately 2,000 , the lowest number.

We next examine whether there is a relationship between $\mathrm{N}^{*}\left(\mathrm{~N}^{* *}\right)$ and the incremental explanatory power that the MVI provides for the ten studies whose main regression we replicate. To do this, we rely on results from Table 4: the incremental explanatory power of the MVI above that of fixed effects. ${ }^{14}$ The rank correlation between incremental explanatory power and $\mathrm{N}^{*}\left(\mathrm{~N}^{* *}\right)$ is $-0.436(-0.423)$. This provides evidence that $\mathrm{N}^{*}$ and $\mathrm{N}^{* *}$ can serve as indicators of the extent to which the MVI helps to explain the dependent variable.

Since $\mathrm{N}^{*}$ is truly simple to calculate for both authors and readers, we believe that the insights provided by this rule-of-thumb metric is worth the modest effort. $\mathrm{N}^{*}$ can act as a complement to t-statistics to get a better sense of variables' incremental explanatory power and also as an antidote to the arbitrary research convention of generally disregarding $\mathrm{N}$ to arrive at conclusions.

\footnotetext{
${ }^{14}$ In other words, we compare Pearson correlations between the dependent variable and fitted values based only fixed effects to Pearson correlations between the dependent variable and fitted values based on fixed effects and the main variable of interest.
} 


\section{6 - Standardized Regressions}

Since t-statistics do not adequately inform about variables' incremental explanatory power, this section addresses whether there is a better way of assessing regression outcomes. The key idea is to move away from a reliance on t-statistics and to instead consider the magnitudes of the coefficient estimates. The question we aim to answer is: how can an OLS-based regression provide evidence on an independent variable's incremental explanatory power, without referring to tstatistics? We hence seek to provide an alternative to calculating changes in $\mathrm{R}^{2}$, discussed earlier. Ideally, such an alternative would not provide mixed signals (such as statistically significant variables without incremental explanatory power).

We propose and evaluate Standardized Regressions, SR, a well-established technique that goes back at least 50 years (examples of usage in the accounting literature include Hirst 1983; Ferreira and Matos 2008; Shan, Taylor, and Walter 2014). SR regressions estimate the coefficients after a normalization of all variables (including the dependent variable) to ensure unit standard deviation. The absolute value of estimated coefficients, generally referred to as Abs. Beta, can loosely be thought of as partial correlation coefficients. As discussed in Section 2, absent multicollinearity, estimated coefficients show the relative importance among independent variables; the coefficients equal the simple correlations between the dependent and each independent variable. The absolute value of estimated coefficients can hence be compared across different studies, just like ordinary correlations can be compared across settings—in sharp contrast to t-statistics. ${ }^{15}$

\footnotetext{
${ }^{15} \mathrm{SR}$ can be viewed as an easier to interpret concept of materiality than notions of "economic significance". The latter involves more subjectivity insofar that the elasticity analysis has to translate into an effect on the dependent variable which then has to be assessed. It is clear that such analyses can be exceedingly sensitive to the specifics of the regression model. That is, changes of the right-hand side (and the specification of the dependent variable, too) can influence the coefficient estimated and the related one-standard-deviation effect. Studies that report on the economic significance of the MVI always find it to be material. Furthermore, "economic significance" does not allow for interstudy effect comparisons. In or view, if one acknowledges the lack of robustness and excess subjectivity related to this method, SR is a natural replacement.
} 
The suggestion that SR coefficients connect with incremental explanatory power rests on its intuitive appeal. As noted earlier, in the presence of multicollinearity, the literature does not back the claim with solidly grounded precepts or formal analyses (see Bring (1994) for extensions and limits). Even absent such a theoretical framework, one can investigate whether contextual empirical evidence supports the proposition that SR coefficients inform on variables' incremental explanatory power. Our main object pertains to identifying a minimum value of the estimated coefficient, below which the incremental explanatory power provided by the variable is effectively immaterial.

Using our ten replicated studies, we evaluate whether SR coefficients connect with incremental explanatory power, measured as the change in Pearson correlation between the dependent variable and fitted model values. For each study, we first calculate the Pearson correlation between the dependent variable and fitted values using all independent variables. We then remove one variable at a time (with replacement) and calculate the Pearson correlation again. ${ }^{16}$ The difference between these two Pearson correlations is denoted Chg. in Corr. It measures each variable's incremental explanatory power and can be analyzed in conjunction with each variable's SR coefficient, $A b s$. Beta in SR. We obtain a total of 176 pairs of Chg. in Corr and Abs. Beta in SR across all ten studies. Figure 3 plots these 176 pairs. Given the motivation related to the use of SR, a first step is to evaluate whether a distinct positive relation between Chg. in Corr and Abs. Beta in SR exists.

\section{Figure 3, Panel A. Standardized regression coefficients}

The plot in Figure 3, Panel A shows that Abs. Beta in SR and Chg. in Corr. associate positively: the Spearman correlation is 0.914 . In other words, a variable's SR coefficient relates to its

\footnotetext{
${ }^{16}$ If there are $\mathrm{K}$ independent variables, we hence obtain $\mathrm{K}$ correlations.
} 
incremental explanatory power in a distinct way; small (large) estimated SR coefficients generally occur when variables' incremental explanatory power is small (large). We underscore that this conclusion holds even though the 176 pairs of Chg. in Corr and Abs.Beta in SR plotted derive from ten vastly different regression specifications and settings.

\section{Figure 3, Panel B. Standardized regression coefficients (zoomed)}

Next, we explore whether a rule of thumb exists such that Abs. Beta in $S R$ can act as a diagnostic for variable materiality. Figure 3, Panel B displays a zoomed-in version of the scatterplot in Panel A: it focuses on small values of the estimated SR coefficients. This panel shows that for cases in which Abs. Beta in SR is smaller than 0.05, Chg. in Corr is almost exclusively smaller than 0.001 . Our proposed rule of thumb is therefore that if a Standardized Regression coefficient is smaller than 0.05 , then the associated independent variable is effectively without incremental explanatory power. If the objective is to identify a parsimonious model to effectively explain the dependent variable, such independent variables might as well be deleted.

We conclude this section by again stressing that interpreting results from our data analysis introduces subjectivity, in particular regarding what constitutes material incremental explanatory power. If anything, we believe that the proposed cutoff 0.05 as a practical rule of thumb is conservative (Figure 3, Panel B shows that a cut-off of twice the magnitude, 0.1, does not seem unreasonable). No matter the subjectivity of cut-off values, a Standardized Regression analysis will inform readers who care about the relative explanatory power of the independent variables.

While the use of Standardized Regressions may not be the panacea to resolve the issue of variable materiality, it helpfully illustrates our point that the current empirical accounting research paradigm has become too simplistic in its overreliance on classical statistics. To presume that a 
significant t-statistic is necessary and sufficient to support a conclusion would seem to make little sense if one allows for the possibility that $\mathrm{N}$ acts as the key driver in a null hypothesis rejection. Standardized Regression, by contrast, avoids this fallacy.

\section{Summary}

Current accounting research practice reaffirms the entrenched status of classical statistics in general, and the attention given to t-statistics in particular. To challenge such well-established practice rekindles a lost battle. In our view, its pervasiveness implies that it cannot simply be dismissed, regardless of its drawbacks (for a persuasive counter-argument, see McShane et al. 2019). Motivated by the findings in this paper, our perspective is more qualified: we suggest expanded analyses to mitigate what we perceive as weaknesses of current research conventions. It is well to note that there are no practical difficulties preventing the implementation of such expanded analyses.

We believe that expanded data analyses ultimately are inevitable. Paradoxically, as research narratives become more and more elaborate, supporting statistical evidence seems to have become less rigorous. Any disparity between narratives and statistical evidence can cynically be interpreted as a deliberate attempt by the researcher(s) to avoid mentioning facts that run counter to the soughtafter rejection of a null hypothesis. As the field matures and gains more experience in handling large datasets and inquiries for which there are no clear-cut answers, we predict that researchers will become more comfortable with the notion that conclusions are more fluid and subjective than they have been in the past. In this regard, we agree that expanded analyses can affect conclusions and, in most cases, they introduce subjectivity in how to interpret the totality of evidence. We emphasize that subjectivity and the need for judgement in interpreting results should not be viewed 
as something negative. Research practice should recognize that no modes of analyses can guarantee a clear "Yes" or "No" answer, especially not to an elaborate inquiry that does not concern first-order phenomena. The real world is inherently complex, often too complex to allow for the reaching such an ambitious research goal.

If one grants the need for additional data analyses and reporting, this paper offers four straightforward suggestions:

1. There is no good reason why papers should not restore the past practice of reporting how the goodness-of-fit of a model improves once the main variable of interest is added to the regression's right-hand side. Aside from changes in $\mathrm{R}^{2}$, to avoid the problem of statistical overfitting, one may also consider a change to an independent evaluation criterion, such as rank correlations, or some other metric suitable given the problem at hand. Since the change in goodness-of-fit is primarily of descriptive importance, there should be no need for an F-statistic to test the null hypothesis of no improvement. Readers should be allowed to form their own judgement as to the materiality of the improvement. It may well be contextual and require additional analyses.

2. The researcher should report on the results of Standardized Regressions, with specific emphasis on the magnitude of the coefficient related to the main variable of interest. The reader may want to reflect on whether this coefficient's magnitude ought to be viewed as marginal, as opposed to material or even compelling. If the main variable of interest ends up with a coefficient smaller than 0.05 , the reader would be well served if the paper explains why the variable should nonetheless be viewed as important.

3. The researcher can help readers understand results by clearly explicating which variables are the most important to explain the dependent variable (beyond references to prior 
literature). It sets the stage for a discussion of the role of the controlling variables in the regression specification.

4. How important is the sample size to achieve null hypothesis rejection? Can one reasonably argue that, within the scheme of accounting research, it is not exceedingly large? Ideally, the paper would report something similar to the $\mathrm{N}^{* *}$ metric proposed in this paper, but even the simplistic back-of-the-napkin metric $\mathrm{N}^{*}$ would inform the reader about the sample size impact as it relates to the paper's conclusions.

Implementing the above suggestions serves the broad purpose of allowing conscientious researchers to signal that they want to avoid false rejections of the null hypothesis. Even more important, in our view, is that a researcher would demonstrate an awareness that many readers may want to make up their own minds about the extent to which the data support the research hypothesis considered. This observation obviously acts a punchline: it leads to the imperative that data analysis and the supply of statistical results ought to go beyond current conventions. 


\section{References}

Abadie, A. 2020. Statistical Nonsignificance in Empirical Economics. American Economic Review: Insights, 2 (2): 193-208.

Afifi, A. A., and Clarke, V. 1990. Computer Aided Multivariate Analysis (2nd ed.), New York Van Nostrand Reinhold.

Andrews, I., and Kasy, M. 2019. Identification of and Correction for Publication Bias. American Economic Review 109 (8): 2766-94.

Black, B. S., Desai, H., Litvak, K., Yoo, W., and Yu, J. J. 2019. Pre-analysis plan for the reg sho reanalysis project. Available at SSRN 3415529.

Biddle, G. C., Seow, G. S., and Siegel. A. F. 1995. Relative versus Incremental Information Content. Contemporary Accounting Research 12 (1): 1-23.

Bring, J. 1994. How to standardize regression coefficients. The American Statistician 48 (3): 209213.

Brodeur, A., Lé, M., Sangnier, M. and Zylberberg, Y. 2016. Star wars: The empirics strike back. Applied Economics 8 (1): 1-32.

Chordia, T., Goyal, A., and Saretto, A. 2017. p-hacking: Evidence from two million trading strategies. Swiss Finance Institute Research Paper, (17-37).

Darlington, R. B. 1990. Regression and linear models. New York: McGraw-Hill.

Dyckman, T. R. and Zeff, S. A. 2014. Some methodological deficiencies in empirical research articles in accounting. Accounting Horizons 28 (3): 695-712.

Dyckman, T. R. and Zeff, S. A. 2015. Accounting research: past, present, and future. Abacus 51 (4): 511-524.

Dyckman, T. R. 2016. Significance Testing: We Can Do Better. Abacus 52 (2): 319-342. 
Fama, E., and MacBeth, J. 1973. Risk, Return and Equilibrium: Empirical Tests. Journal of Political Economy 81:607-36.

Ferreira, M. A., and Matos, P. 2008. The colors of investors' money: The role of institutional investors around the world. Journal of Financial Economics 88(3): 499-533.

Gannon, M. A., de Bragança Pereira, C. A., and Polpo, A. 2019. Blending Bayesian and classical tools to define optimal sample-size-dependent significance levels. The American Statistician 73(sup1): 213-222.

Gelman, A., and Stern, H. 2006. The Difference between 'Significant' and 'Not Significant' Is Not Itself Statistically Significant. American Statistician 60 (4): 328-31.

Greene, W. H. 2003. Econometric analysis. Pearson Education India.

Harvey, C. R. 2017. Presidential address: The scientific outlook in financial economics. The Journal of Finance 72 (4): 1399-1440.

Harvey, C.R., Liu, Y. and Zhu, H. 2016. ... and the cross-section of expected returns. The Review of Financial Studies 29 (1): 5-68.

Hail, L., Lang, M., and Leuz, C. 2020. Reproducibility in Accounting Research: Views of the Research Community. Journal of Accounting Research 58 (2): 519-543.

Hirst, M. K. 1983. Reliance on accounting performance measures, task uncertainty, and dysfunctional behavior: Some extensions. Journal of Accounting Research 596-605.

Ioannidis, J. P. A. 2005. Why most published research findings are false. PLoS medicine 2 (8): 124.

Ioannidis, J. P. A., Stanley, T. D. and Doucouliagos, H. 2017. The Power of Bias in Economics Research. Economic Journal 127 (605): F236-65.

Kim, J. H., Ahmed, K., and Ji, P. I. 2018. Significance testing in accounting research: a critical evaluation based on evidence. Abacus 54 (4): 524-546. 
Leamer, E. 1978. Specification Searches: Ad Hoc Inferences with Nonexperimental Data, Wiley, New York.

Leone, A. J., Minutti-Meza, M., and Wasley, C. E. 2019. Influential observations and inference in accounting research. The Accounting Review 94 (6): 337-364.

Lindley, D. V. 2014. Understanding uncertainty. Revised Edition, Wiley.

McShane, B. B., Gal, D., Gelman, A, Robert, C., and Tackett, J. L. 2019. Abandon Statistical Significance. American Statistician 73 (S1): 235-45.

Moosa, I. A. 2017. Econometrics as a con art: exposing the limitations and abuses of econometrics. Edward Elgar, Cheltenham.

Ohlson, J. A. 2015. Accounting research and common sense. Abacus 51 (4): 525-535.

Ohlson, J. A. 2020. Researchers' data analysis choices: An excess of false positives? The Review of Accounting Studies, Forthcoming.

Petersen, M. A. 2009. Estimating standard errors in finance panel data sets: Comparing approaches. The Review of Financial Studies 22 (1): 435-480.

Powell, J., Shi, J., Smith, T. and Whaley, R. 2009. Common divisors, payout persistence, and return predictability. International Review of Finance 9 (4):335-357.

Shan, Y., Taylor, S., and Walter, T. 2014. The role of "other information" in analysts' forecasts in understanding stock return volatility. Review of Accounting Studies 19 (4): 1346-1392.

Silver, N. 2012. The signal and the noise: why so many predictions fail-but some don't. Penguin.

Spanos, A. 2013. Who should be afraid of the Jeffreys-Lindley paradox? Philosophy of Science 80 (1): 73-93.

Walsh, A. 1990. Statistics for the social sciences. New York: Harper \& Row. 


\section{Exhibits}

\section{Table 1. Characterization of the ten original studies}

Table 1 shows absolute $t$-statistics, adj. $\mathrm{R}^{2}$, and number of observations for our sample of 10 replicated studies. All variables are anonymized and denoted Varl - Var31. The variables are sorted according to descending t-statistics. The absolute value of the $\mathrm{t}$-statistic for the main variable of interest in each study is in boldface.

\begin{tabular}{|c|c|c|c|c|c|c|c|c|c|c|}
\hline \multirow[b]{2}{*}{ Studies } & \multicolumn{10}{|c|}{ Absolute T } \\
\hline & 1 & 2 & 3 & 4 & 5 & 6 & 7 & 8 & 9 & 10 \\
\hline Var1 & 29.xx & 10.xx & 11.xx & 18.xx & 38.xx & 56.xx & 253.xx & 16.xx & 73.xx & 11.xx \\
\hline Var2 & 22.xx & 3.xx & 11.xx & 16. $x x$ & 14.xx & 16.xx & 31.xx & 13.xx & 14.xx & 10.xx \\
\hline Var3 & 11.xx & 2.xx & 9.xx & 10.xx & 13.xx & 7.xx & 28.xx & 12.xx & 11.xx & 9.xx \\
\hline Var4 & 8.xx & 2.xx & 8.xx & 9.xx & 12.xx & $6 . x x$ & 28.xx & 11.xx & 9.xx & 8.xx \\
\hline Var5 & 8.xx & 1.xx & 7.xx & 9.xx & 10.xx & 5.xx & 21.xx & 10.xx & 8.xx & $6 . x x$ \\
\hline Var6 & 7.xx & 1.xx & 5.xx & $6 . x x$ & 7.xx & $5 . x x$ & 20.xx & 7.xx & 8.xx & $6 . x x$ \\
\hline Var7 & 5.xx & 1.xx & 4. $x x$ & $5 . x x$ & 7.xx & 4. $x x$ & 15.xx & 7.xx & 7.xx & $6 . x x$ \\
\hline Var8 & $3 . x x$ & 1.xx & 4. $x x$ & 5.xx & 7.xx & $3 . x x$ & $9 . x x$ & $6 . x x$ & $6 . x x$ & $5 . x x$ \\
\hline Var9 & 3.xx & 1.xx & 4. $x x$ & 5.xx & $6 . x x$ & 3.xx & 8.xx & 5.xx & 5.xx & 4. $\mathrm{xx}$ \\
\hline Var10 & 1.xx & $0 . x x$ & 3.xx & 4. $x x$ & 5.xx & 2.xx & $6 . x x$ & 5.xx & 4. $x x$ & 4. $\mathrm{xx}$ \\
\hline Var11 & 1.xx & $0 . x x$ & 2.xx & 4. $x x$ & 4. $x x$ & 2.xx & 4. $x x$ & 4. $x x$ & 4.xx & 4.xx \\
\hline Var12 & & $0 . x x$ & 2.xx & 3.xx & 4. $\mathrm{xx}$ & 2.xx & 4. $\mathrm{xx}$ & 4.xx & 3.xx & 4. $x x$ \\
\hline Var13 & & $0 . x x$ & 2.xx & 2.xx & 3.xx & 1.xx & 3. $x x$ & 3.xx & 3. $x x$ & 4. $x x$ \\
\hline Var14 & & & $0 . x x$ & 2.xx & 3. $x x$ & $0 . x x$ & 3. $x x$ & 3.xx & 3.xx & 3. $x x$ \\
\hline Var15 & & & & 2.xx & 3.xx & $0 . x x$ & 3.xx & 2.xx & 2.xx & 3. $x x$ \\
\hline Var16 & & & & $0 . x x$ & 2.xx & $0 . x x$ & 1.xx & 2.xx & $2 . x x$ & 3.xx \\
\hline Var17 & & & & & & $0 . x x$ & $0 . \mathrm{xx}$ & 2.xx & 1.xx & 3. $x x$ \\
\hline Var18 & & & & & & & $0 . \mathrm{xx}$ & $0 . \mathrm{xx}$ & 1.xx & 3. $x x$ \\
\hline Var19 & & & & & & & & & 1.xx & 3.xx \\
\hline Var20 & & & & & & & & & 1.xx & 2.xx \\
\hline Var21 & & & & & & & & & 1.xx & 2.xx \\
\hline Var22 & & & & & & & & & $0 . x x$ & 1.xx \\
\hline Var23 & & & & & & & & & & 1.xx \\
\hline Var24 & & & & & & & & & & 1.xx \\
\hline Var25 & & & & & & & & & & 1.xx \\
\hline Var26 & & & & & & & & & & 1.xx \\
\hline Var27 & & & & & & & & & & 1.xx \\
\hline Var28 & & & & & & & & & & 1.xx \\
\hline Var29 & & & & & & & & & & 1.xx \\
\hline Var30 & & & & & & & & & & $0 . x x$ \\
\hline Var31 & & & & & & & & & & $0 . x x$ \\
\hline Adj. $R^{2}$ & $0.7 x x$ & $0.04 \mathrm{x}$ & $0.03 x$ & $0.5 \mathrm{xx}$ & $0.8 \mathrm{xx}$ & $0.8 \mathrm{xx}$ & $0.5 \mathrm{xx}$ & $0.3 \mathrm{xx}$ & $0.8 \mathrm{xx}$ & $0.3 \mathrm{xx}$ \\
\hline $\mathrm{N}$ & $20 \mathrm{xxx}$ & $16 \mathrm{xxx}$ & $56 \mathrm{xxx}$ & $14 \mathrm{xxx}$ & $19 \mathrm{xxx}$ & $11 \mathrm{xxx}$ & $2471 x x x$ & $26 \mathrm{xxx}$ & 19xxx & $41 \mathrm{xxx}$ \\
\hline
\end{tabular}


Table 2. Success of the ten replications

Table 2 shows the absolute $\mathrm{t}$-statistics, adj. $\mathrm{R}^{2}$, and number of observations for our sample of 10 replicated studies. Juxtaposed to Table 1, this table shows how successfully the replications were. All variables are anonymized and denoted Varl - Var31. The variables are sorted according to descending t-statistics. The absolute value of the t-statistic for the main variable of interest in each study is in boldface. Correct sign, significant vars. (Correct sign, insignificant vars.) shows the number of correct signs (i.e. the same sign as in the original study) for significant (insignificant) variables. $N$ Diff is the difference in sample size between relative to the sample size of the original studies. Adj. $R^{2}$ Diff shows the difference in adjusted $\mathrm{R}^{2}$ between the replicated and original studies.

\begin{tabular}{|c|c|c|c|c|c|c|c|c|c|c|}
\hline \multirow[b]{2}{*}{ Studies } & \multicolumn{10}{|c|}{ Absolute T } \\
\hline & 1 & 2 & 3 & 4 & 5 & 6 & 7 & 8 & 9 & 10 \\
\hline Var1 & 24.89 & 7.13 & 14.98 & 18.86 & 47.18 & 53.61 & 150.71 & 10.44 & 67.74 & 9.67 \\
\hline Var2 & 17.31 & 3.67 & 13.36 & 16.34 & 15.90 & 13.02 & 22.39 & 8.94 & 11.67 & 7.96 \\
\hline Var3 & 10.61 & 2.39 & 12.21 & 10.80 & 15.42 & 7.11 & 22.20 & 8.73 & 9.89 & 7.47 \\
\hline Var4 & 9.27 & 2.35 & 11.41 & 9.30 & 12.64 & 6.75 & 20.43 & 6.96 & 9.73 & 5.99 \\
\hline Var5 & 8.59 & 1.86 & 10.29 & 7.19 & 11.59 & 6.61 & 20.09 & 6.88 & 8.50 & 5.53 \\
\hline Var6 & 7.89 & 1.56 & 9.87 & 6.16 & 11.06 & 6.30 & 19.78 & 6.43 & 8.35 & 5.09 \\
\hline Var7 & 7.73 & 1.32 & 7.29 & 5.33 & 9.48 & 6.27 & 9.05 & 6.02 & 7.33 & 5.02 \\
\hline Var8 & 5.87 & 1.20 & 6.57 & 5.14 & 9.20 & 3.65 & 8.29 & 5.14 & 7.17 & 4.97 \\
\hline Var9 & 4.95 & 1.20 & 4.99 & 4.15 & 6.74 & 3.43 & 7.95 & 4.80 & 7.11 & 4.85 \\
\hline Var10 & 4.49 & 0.88 & 4.52 & 3.17 & 6.62 & 2.92 & 7.89 & 3.97 & 5.70 & 4.83 \\
\hline Var11 & 2.19 & 0.69 & 4.48 & 2.98 & 5.23 & 2.26 & 7.53 & 3.70 & 5.61 & 4.36 \\
\hline Var12 & & 0.39 & 3.29 & 2.89 & 5.03 & 1.54 & 5.06 & 3.42 & 4.44 & 4.17 \\
\hline Var13 & & 0.28 & 2.61 & 2.09 & 3.87 & 1.16 & 5.02 & 2.29 & 3.23 & 4.14 \\
\hline Var14 & & & 2.04 & 1.95 & 3.63 & 1.11 & 4.23 & 1.97 & 2.72 & 4.00 \\
\hline Var15 & & & & 1.62 & 2.32 & 1.02 & 3.29 & 1.30 & 2.45 & 3.54 \\
\hline Var16 & & & & 1.01 & 0.43 & 0.88 & 1.51 & 0.80 & 1.75 & 3.35 \\
\hline Var17 & & & & & & 0.10 & 0.91 & 0.50 & 1.55 & 3.17 \\
\hline Var18 & & & & & & & 0.38 & 0.15 & 1.34 & 3.15 \\
\hline Var19 & & & & & & & & & 1.05 & 3.12 \\
\hline Var20 & & & & & & & & & 0.94 & 3.05 \\
\hline Var21 & & & & & & & & & 0.41 & 2.77 \\
\hline Var22 & & & & & & & & & 0.15 & 2.71 \\
\hline Var23 & & & & & & & & & & 2.16 \\
\hline Var24 & & & & & & & & & & 2.06 \\
\hline Var25 & & & & & & & & & & 1.87 \\
\hline Var26 & & & & & & & & & & 1.68 \\
\hline Var27 & & & & & & & & & & 1.68 \\
\hline Var28 & & & & & & & & & & 0.97 \\
\hline Var29 & & & & & & & & & & 0.38 \\
\hline Var30 & & & & & & & & & & 0.31 \\
\hline Var31 & & & & & & & & & & 0.12 \\
\hline Adj. $R^{2}$ & 0.734 & 0.019 & 0.060 & 0.496 & 0.827 & 0.773 & 0.528 & 0.264 & 0.880 & 0.366 \\
\hline $\mathrm{N}$ & 20216 & 16061 & 53618 & 14922 & 19118 & 11331 & 2360124 & 28002 & 18727 & 41100 \\
\hline
\end{tabular}




\begin{tabular}{lcccccccccc}
\hline $\begin{array}{l}\text { Correct sign, } \\
\text { significant vars. }\end{array}$ & $9 / 9$ & $5 / 7$ & $13 / 13$ & $15 / 15$ & $16 / 16$ & $12 / 12$ & $15 / 15$ & $16 / 17$ & $15 / 16$ & $25 / 25$ \\
\hline $\begin{array}{l}\text { Correct sign, } \\
\text { insignificant. vars. }\end{array}$ & $2 / 2$ & $4 / 6$ & $1 / 1$ & $1 / 1$ & $0 / 0$ & $2 / 5$ & $3 / 3$ & $1 / 1$ & $3 / 6$ & $5 / 6$ \\
\hline \multicolumn{1}{|c}{ Adj. $\mathrm{R}^{2}$ Diff. } & -0.017 & -0.025 & 0.024 & -0.030 & 0.017 & -0.036 & -0.032 & -0.062 & 0.013 & 0.027 \\
\hline N Diff. & -0.028 & -0.015 & -0.054 & 0.000 & -0.017 & -0.034 & -0.045 & 0.046 & -0.032 & -0.013 \\
\hline
\end{tabular}




\section{Table 3. Correlations: main variables of interest and dependent variables}

Table 3 shows Pearson and Spearman correlations between the main variable of interest and the dependent variable for each replicated study. The correlations derive from data pertaining to our replications, not the original studies.

\begin{tabular}{lcccccccccc}
\hline & \multicolumn{10}{c}{ Studies } \\
\hline & 1 & 2 & 3 & 4 & 5 & 6 & 7 & 8 & 9 & 10 \\
\hline P. Corr. & 0.119 & 0.026 & 0.000 & 0.174 & -0.008 & 0.347 & -0.008 & -0.297 & 0.046 & -0.098 \\
S. Corr. & 0.152 & 0.025 & 0.011 & 0.160 & -0.007 & 0.400 & -0.011 & -0.214 & 0.050 & -0.293 \\
\hline
\end{tabular}




\section{Table 4. Correlations: main variables of interest, fixed effects, and dependent variables}

Table 4 shows Pearson correlations between dependent variables and two sets of fitted values. FE bivariate denotes Pearson correlations between the dependent variable and fitted values based solely on fixed effects. Similarly, $F E+M V I$ bivariate denotes Pearson correlations between the dependent variable and fitted values based on fixed effects plus the main variable of interest. Chg. in Corr denotes the difference in Pearson correlation between these two specifications. P. Corr. (Full Model) denotes Pearson correlations between the dependent variable and fitted values based on all regressors in each study. The correlations derive from data pertaining to our replications, not the original studies.

\begin{tabular}{lcccccccccccc}
\hline & \multicolumn{1}{c}{ Studies } \\
\hline & 1 & 2 & 3 & 4 & 5 & 6 & 7 & 8 & 9 & 10 \\
\hline FE bivariate & 0.344 & 0.157 & 0.126 & 0.551 & 0.448 & 0.506 & 0.230 & 0.282 & 0.408 & 0.087 \\
FE+MVI bivariate & 0.367 & 0.163 & 0.126 & 0.555 & 0.449 & 0.624 & 0.230 & 0.396 & 0.409 & 0.131 \\
Chg. in Corr & 0.023 & 0.005 & 0.000 & 0.004 & 0.000 & 0.118 & 0.000 & 0.114 & 0.001 & 0.044 \\
\hline & & & & & & & & & & & \\
P. Corr. (Full Model) & 0.863 & 0.181 & 0.248 & 0.710 & 0.910 & 0.880 & 0.727 & 0.516 & 0.939 & 0.606 \\
\hline
\end{tabular}




\section{Table 5. Main variables of interest and residuals from the baseline models}

Table 5 shows Pearson (Spearman) correlations between the main variable of interest and the residuals from the baseline model. "Baseline model" denotes the original model specification exclusive of the main variable of interest. P. Corr. (S. Corr.). denotes Pearson (Spearman) correlations. Control vars. indicates the number of control variables. The correlations derive from data pertaining to our replications, not the original studies.

\begin{tabular}{lcccccccccc}
\hline & & \multicolumn{1}{c}{ Studies } \\
\hline & 1 & 2 & 3 & 4 & 5 & 6 & 7 & 8 & 9 & 10 \\
\hline P. Corr. & 0.076 & 0.021 & -0.010 & 0.045 & -0.029 & 0.031 & -0.006 & -0.051 & -0.036 & -0.012 \\
P-value & 0.000 & 0.008 & 0.017 & 0.000 & 0.000 & 0.001 & 0.000 & 0.000 & 0.000 & 0.016 \\
S. Corr. & 0.076 & 0.019 & -0.010 & 0.053 & -0.028 & 0.029 & -0.004 & 0.028 & -0.033 & 0.024 \\
P-value & 0.000 & 0.017 & 0.020 & 0.000 & 0.000 & 0.002 & 0.000 & 0.000 & 0.000 & 0.000 \\
N & 20216 & 16061 & 53618 & 14922 & 19118 & 11331 & 2360124 & 28002 & 18727 & 41100 \\
Control vars. & 10 & 12 & 13 & 15 & 15 & 16 & 17 & 17 & 21 & 30 \\
\hline
\end{tabular}


Table 6. Incremental explanatory power of the main variables of interest

Table 6 shows Pearson (Spearman) correlations between the dependent variable and fitted values from i) the full model, and ii) from the baseline model. "Full" denotes the original model specification. "Baseline" denotes the original model specification exclusive of the main variable of interest. P. Corr. (S. Corr.). denotes Pearson (Spearman) correlations. Chg. in P. Corr. (Chg. in S. Corr.) shows the difference in Pearson (Spearman) correlations between the full model and the baseline model. Ind. vars. denotes the number of independent variables in the full model. The correlations derive from data pertaining to our replications, not the original studies

\begin{tabular}{lcccccccccc}
\hline & \multicolumn{10}{c}{ Studies } \\
\hline & 1 & 2 & 3 & 4 & 5 & 6 & 7 & 8 & 9 & 10 \\
\hline P. Corr. (Full) & 0.857 & 0.181 & 0.248 & 0.710 & 0.910 & 0.880 & 0.727 & 0.516 & 0.939 & 0.606 \\
P. Corr. (Baseline) & 0.856 & 0.177 & 0.247 & 0.709 & 0.910 & 0.880 & 0.727 & 0.512 & 0.939 & 0.605 \\
Chg. in P. Corr. & 0.001 & 0.004 & 0.000 & 0.001 & 0.000 & 0.000 & 0.000 & 0.004 & 0.000 & 0.001 \\
\hline S. Corr. (Full) & 0.869 & 0.162 & 0.257 & 0.696 & 0.901 & 0.888 & 0.641 & 0.562 & 0.938 & 0.625 \\
S. Corr. (Baseline) & 0.868 & 0.158 & 0.257 & 0.695 & 0.901 & 0.888 & 0.641 & 0.563 & 0.938 & 0.624 \\
Chg. in S. Corr. & 0.001 & 0.004 & 0.000 & 0.001 & 0.000 & 0.000 & 0.000 & -0.001 & 0.000 & 0.001 \\
\hline N & 20216 & 16061 & 53618 & 14922 & 19118 & 11331 & 2360124 & 28002 & 18727 & 41100 \\
Ind. vars. & 11 & 13 & 14 & 16 & 16 & 17 & 18 & 18 & 22 & 31 \\
\hline
\end{tabular}




\section{Figure 1. Incremental explanatory power by t-statistic (Pearson)}

Figure 1 plots Pearson correlations between the dependent variable and fitted values from regressions equal in number to the number of independent variables, for each firm. The independent variables are sequentially introduced. according to descending absolute t-statistic, for each study. Fixed effects are included in all specifications. The ten studies in our sample are represented by one line each and denoted s1-s10. The correlations derive from data pertaining to our replications, not the original studies.

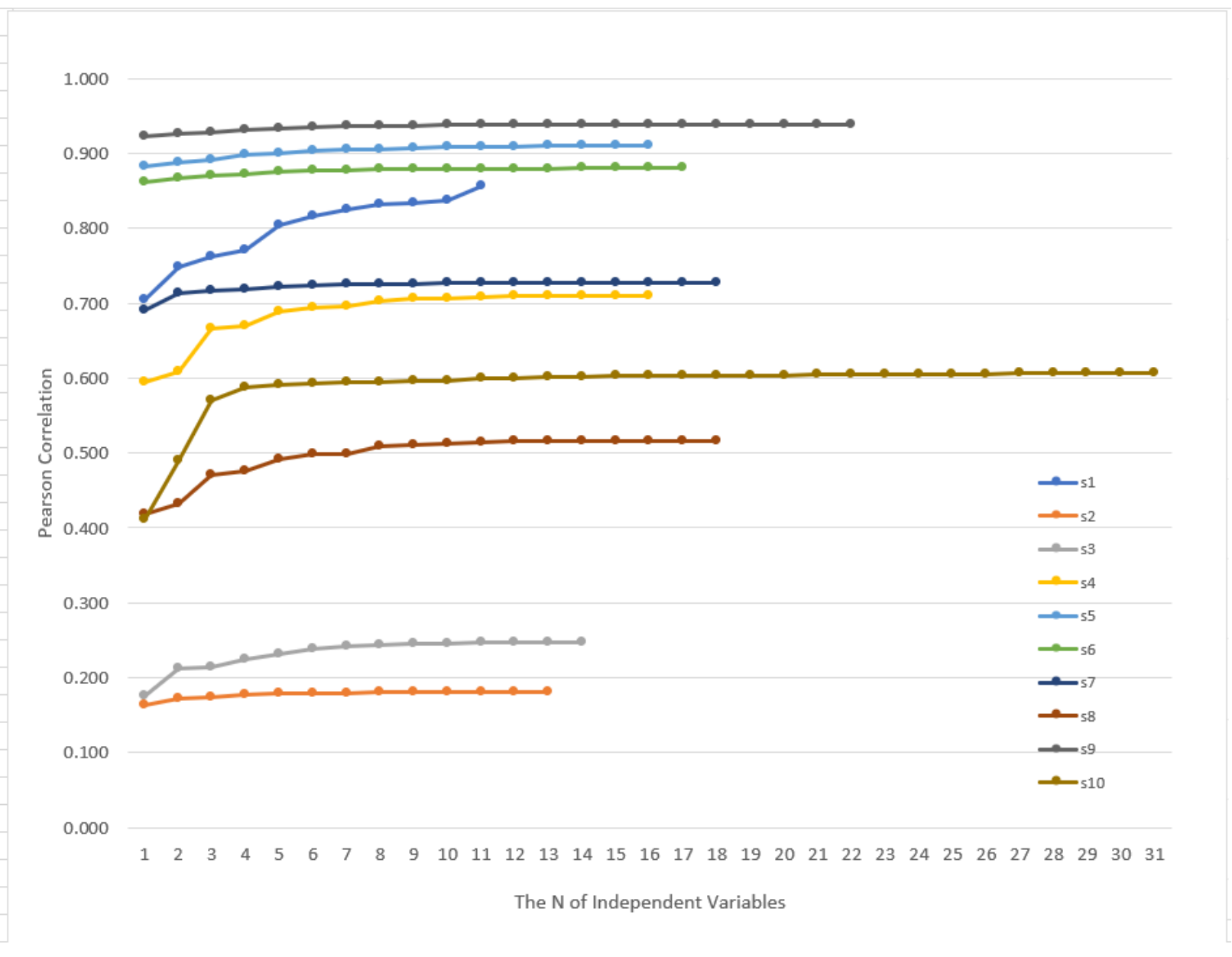




\section{Figure 2. Incremental explanatory power by t-statistic (Spearman)}

Figure 2 plots Spearman correlations between the dependent variable and fitted values from regressions equal in number to the number of independent variables, for each firm. The independent variables are sequentially introduced. according to descending absolute t-statistic, for each study. Fixed effects are included in all specifications. The ten studies in our sample are represented by one line each and denoted s1- s10. The correlations derive from data pertaining to our replications, not the original studies.

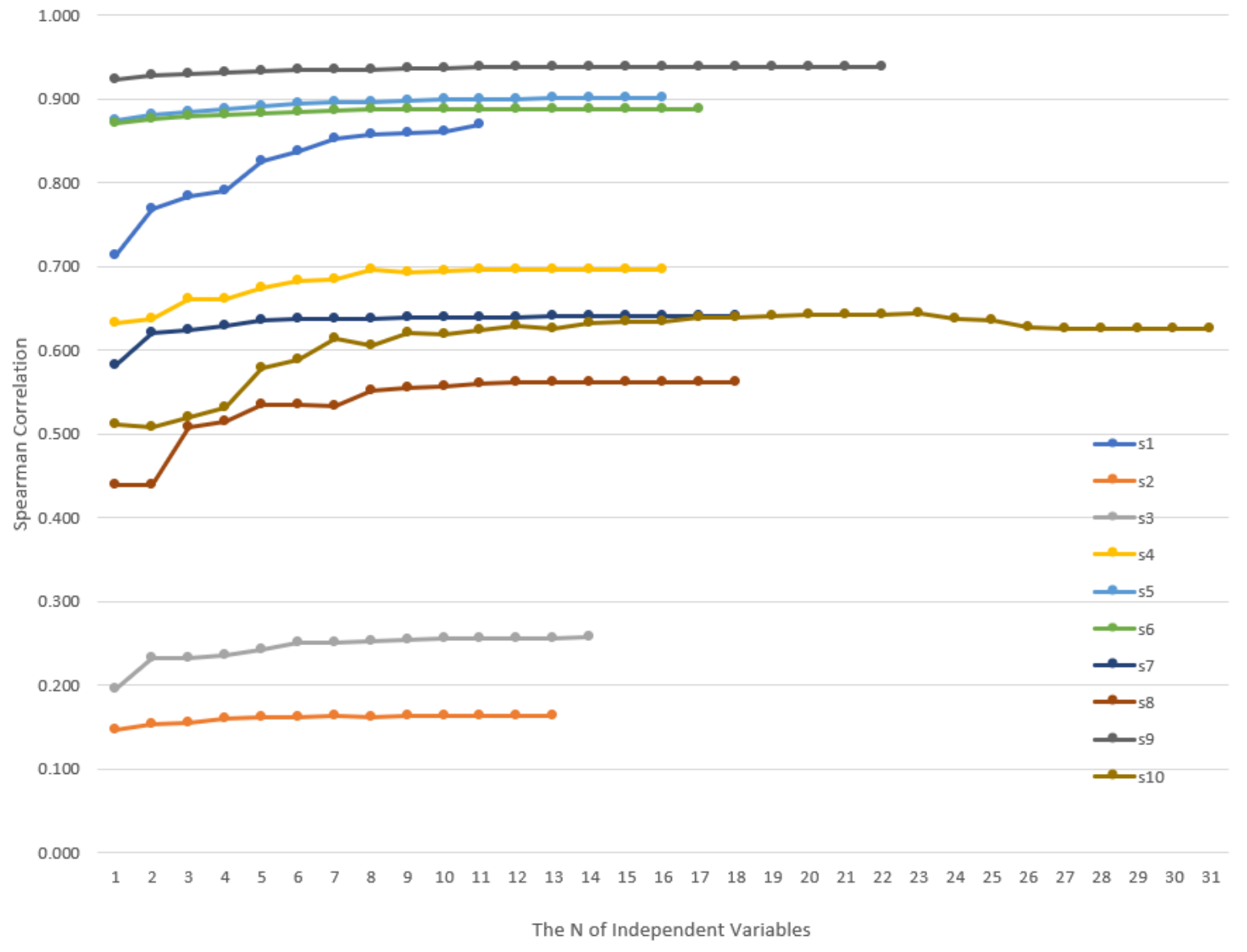




\section{Table 7. $\mathrm{N}^{*}$ and $\mathrm{N} * *$}

Table 7 shows the critical number of observations needed to show a t-statistic of 1.96 for each study. $\mathrm{N}^{*}$ derives from the definition of a t-statistic and is defined as follows: $N^{*}=(2 / t)^{2} \times N . \mathrm{N}^{* *}$ is based on iterative simulations, in which we randomly select datapoints without replacement to form increasingly small subsamples from each replicated study. We run 100 regressions for each subsample size iteration and when the median t-statistic for the main variable interest have an absolute t-statistic that approaches $2, \mathrm{~N}^{* *}$ is obtained. Chg. in Corr denotes the difference between i) the Pearson correlation between the dependent variable and fitted values based solely on fixed effects; and ii) the Pearson correlation between the dependent variable and fitted values based on fixed effects plus the main variable of interest. Abs. $t$ (Full Model) denotes the absolute t-statistic of the main variable of interest from the full model specification. $N$ is the number of observations in the replicated studies. The results derive from data pertaining to our replications, not the original studies.

\begin{tabular}{lccccc}
\hline Studies & $\mathrm{N}$ & $\mathrm{N}^{*}$ & $\mathrm{~N}^{* *}$ & Abs. t (Full Model) & Chg. in Corr. \\
\hline 1 & 20216 & 3164 & 3133 & 4.95 & 0.023 \\
2 & 16061 & 11135 & 11242 & 2.35 & 0.005 \\
3 & 53618 & 30168 & 32170 & 2.61 & 0.000 \\
4 & 14922 & 6473 & 2685 & 2.98 & 0.004 \\
5 & 19118 & 5571 & 5735 & 3.63 & 0.000 \\
6 & 11331 & 8515 & 6798 & 2.26 & 0.118 \\
7 & 2360124 & 354396 & 143967 & 5.06 & 0.000 \\
8 & 28002 & 2599 & 1904 & 6.43 & 0.114 \\
9 & 18727 & 9760 & 3558 & 2.72 & 0.001 \\
10 & 41100 & 15922 & 11097 & 3.15 & 0.044 \\
\hline
\end{tabular}




\section{Figure 3. Standardized regression coefficients}

Figure 3 plots the change in explanatory power, measured as the change in Pearson correlation between the dependent variable and fitted model values. We first calculate the Pearson correlation between the dependent variable and fitted values using all regressors, for each study. We then remove one variable at a time (with replacement) and calculate the Pearson correlations again. The difference between these two Pearson correlations is denoted Chg. in Corr. and shows the incremental explanatory power of each variable. Fixed effects are used in all specifications. This is plotted against the absolute value of variables' standardized regression coefficients (Abs. Beta in SR). Panel A shows all resulting points. Panel B zooms in closer to origin. The results derive from data pertaining to our replications, not the original studies.

Panel A. Full scatterplot

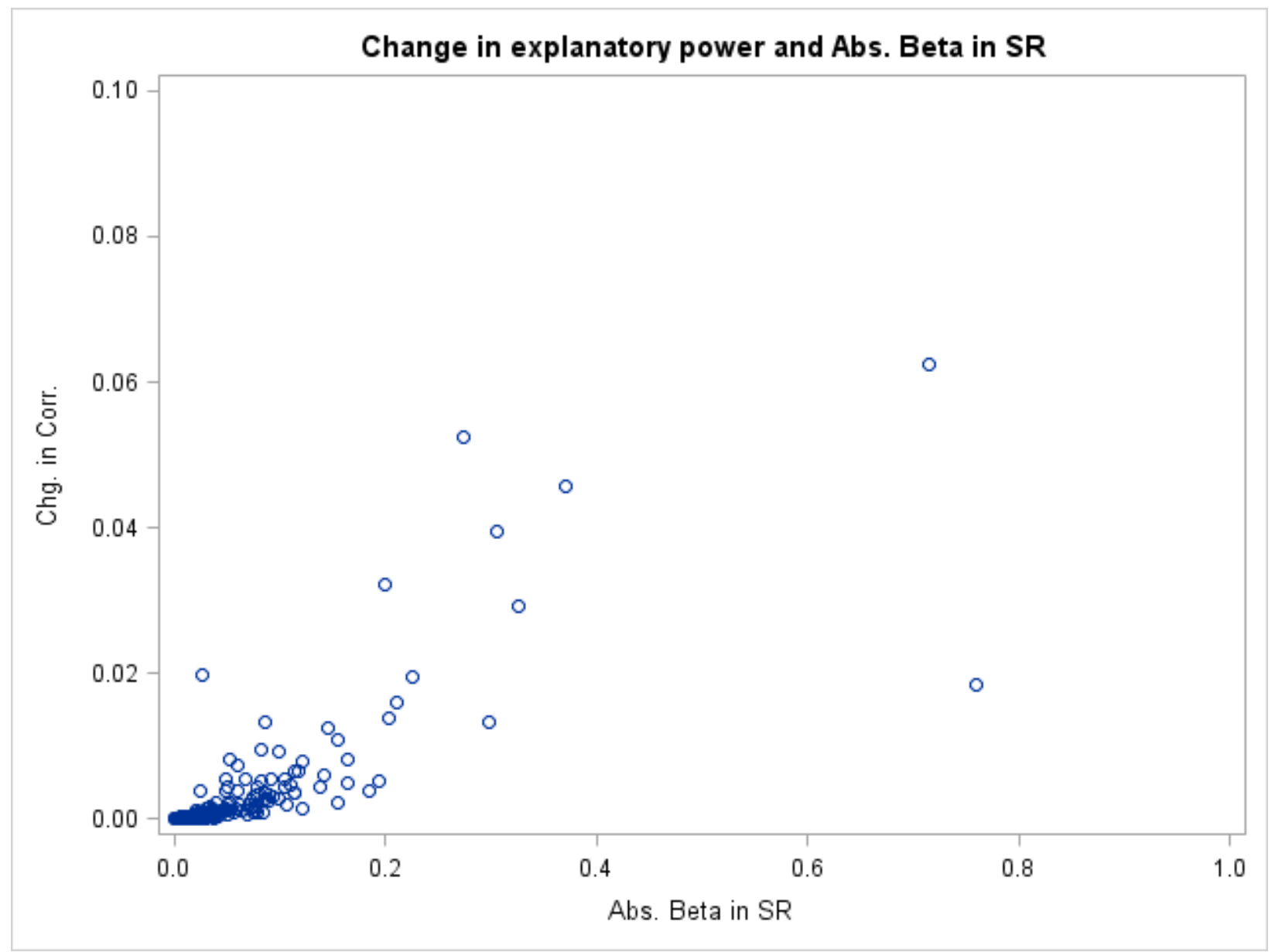


Panel B. Zoomed-in scatterplot

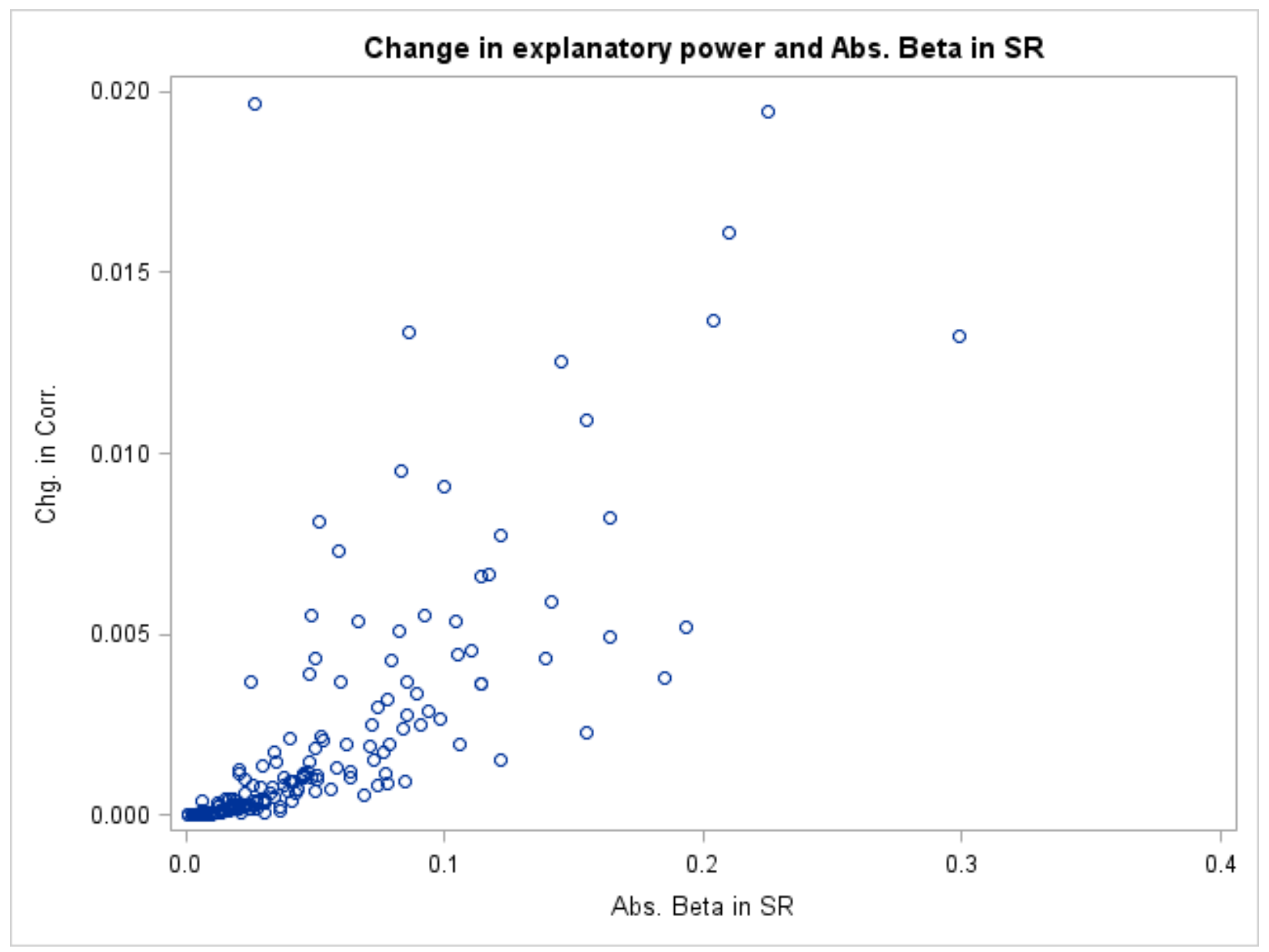


Appendix 1

This table reports results of augmenting the original regression specifications with variables capturing climate information in Denver at the release date of the annual report for each firm-year in each study. Climate data is obtained from https://climate.colostate.edu/data_access.html. Main variables of interest, control variables, and fixed effects are the same as original regressions. Standard errors are clustered in the same level as original studies.

\begin{tabular}{|c|c|c|c|c|c|c|c|c|c|c|}
\hline Studies & 1 & 2 & 3 & 4 & 5 & 6 & 7 & 8 & 9 & 10 \\
\hline \multirow[t]{2}{*}{ Max. temp } & $-0.004 *$ & 0.000 & 0.000 & 0.000 & 0.000 & -0.001 & -0.000 & 0.000 & 0.000 & -0.010 \\
\hline & $(-1.992)$ & $(0.861)$ & $(0.570)$ & $(0.903)$ & $(0.632)$ & $(-1.496)$ & $(-1.252)$ & (1.391) & $(0.533)$ & $(-0.170)$ \\
\hline \multirow[t]{2}{*}{ Min. temp } & $0.005 *$ & -0.001 & 0.000 & $-0.000 *$ & $-0.001 *$ & -0.000 & $0.000 * *$ & -0.000 & -0.000 & -0.067 \\
\hline & $(1.793)$ & $(-0.885)$ & $(0.518)$ & $(-2.234)$ & $(-1.848)$ & $(-0.245)$ & $(2.258)$ & $(-0.488)$ & $(-0.238)$ & $(-1.291)$ \\
\hline \multirow[t]{2}{*}{ Precipitation } & -0.213 & 0.000 & 0.071 & 0.006 & -0.055 & -0.059 & 0.001 & -0.000 & -0.001 & 0.264 \\
\hline & $(-1.238)$ & $(0.007)$ & $(1.540)$ & $(0.504)$ & $(-1.263)$ & $(-1.238)$ & $(0.132)$ & $(-0.001)$ & $(-0.044)$ & $(0.085)$ \\
\hline \multirow[t]{2}{*}{ Snowfall } & 0.005 & 0.008 & 0.002 & 0.000 & -0.001 & 0.003 & -0.000 & -0.001 & -0.004 & -0.411 \\
\hline & $(0.246)$ & $(0.975)$ & $(0.234)$ & $(0.237)$ & $(-0.159)$ & $(0.383)$ & $(-0.241)$ & $(-1.132)$ & $(-1.364)$ & $(-1.094)$ \\
\hline MVI and Controls & Yes & Yes & Yes & Yes & Yes & Yes & Yes & Yes & Yes & Yes \\
\hline Fixed effects & Yes & Yes & Yes & Yes & Yes & Yes & Yes & Yes & Yes & Yes \\
\hline Clustering & Yes & Yes & Yes & Yes & Yes & Yes & Yes & Yes & Yes & Yes \\
\hline \multirow[t]{2}{*}{ Constant } & $3.990 * * *$ & $0.125 * *$ & $-1.782 * * *$ & $0.231 * * *$ & $3.793 * * *$ & $9.712 * * *$ & $0.081 * * *$ & $0.154 * * *$ & $2.974 * * *$ & $28.700 * *$ \\
\hline & (11.187) & $(2.420)$ & $(-15.643)$ & $(12.455)$ & (13.354) & $(104.455)$ & $(23.925)$ & $(9.529)$ & $(32.907)$ & (2.304) \\
\hline $\mathrm{N}$ & 19945 & 15925 & 52084 & 14819 & 19048 & 11276 & 2237362 & 27636 & 18634 & 39348 \\
\hline $\mathrm{R}^{2}$ & 0.735 & 0.032 & 0.062 & 0.505 & 0.828 & 0.775 & 0.532 & 0.267 & 0.882 & 0.365 \\
\hline $\operatorname{adj} . \mathrm{R}^{2}$ & 0.734 & 0.018 & 0.06 & 0.497 & 0.827 & 0.773 & 0.532 & 0.265 & 0.88 & 0.364 \\
\hline
\end{tabular}

